\title{
Complex Variations in Branching Pattern of the Axillary Artery and Hands with the Persistent Median Artery
}

\author{
Jun Hyun Park $\mathbb{D}^{1}$, Se Hyun Kang $\mathbb{D}^{1}$, Tai Kyoung Baik $\mathbb{D}^{1}$, Ran Sook Woo $\mathbb{D}^{1}$, \\ Dae Yong Song $\mathbb{D}^{1, *}$, Hong Il Yoo $\mathbb{D}^{1, *}$ \\ ${ }^{1}$ Department of Anatomy and Neurosciences, Eulji University School of Medicine
}

\begin{abstract}
We report bilateral multiple variations in branching pattern of the axillary artery and superficial palmar arch of the hand in an 84-year-old Korean female cadaver. First, we identified an aberrant trunk with high bifurcation of the deep brachial artery from the left axillary artery. Second, the persistent median artery accompanied with a median nerve and formed the superficial palmar arch in the left hand. Third, a common trunk was originated from the second part of the right axillary artery, and divided into lateral thoracic, subscapular, and circumflex humeral arteries, respectively. Finally, the superficial palmar branch of radial artery lies superficial to the thenar muscles and gave rise to a common trunk of the princeps pollicis and radialis indicis arteries on both hands. This case report alerts clinicians and anatomists to the possibility of concurrent complex bilateral variations in the upper limb.
\end{abstract}

Keywords : Axillary artery, Persistent median artery, Superficial palmar branch of radial artery, Anatomical variations

\section{INTRODUCTION}

The axillary artery is bounded from lateral border of the first rib to lower margin of the teres major muscle and continues as brachial artery. It is divided into three parts by the pectoralis minor and gives off six major branches [1].

This work was supported by Eulji University in 2016, and National Research Foundation of Korea (NRF) Grant funded by the Korea government (MSIT, NRF-2017R1C1B5015910).

*Dae Yong Song and Hong Il Yoo equally contributed as the corresponding authors.

The author(s) agree to abide by the good publication practice guideline for medical journals.

The author(s) declare that there are no conflicts of interest.

Received: November 11, 2020; Revised: November 26, 2020;

Accepted: November 27, 2020

Correspondence to: Hong II Yoo (Department of Anatomy and Neurosciences, Eulji University School of Medicine, Daejeon, Republic of Korea)

E-mail: hiyoo@eulji.ac.kr
In general, the superior thoracic artery is the only branch of the first part. The second part has two branches, the thoracoacromial and lateral thoracic arteries. The third part gives off three branches including the anterior and posterior circumflex humeral and subscapular arteries. Nevertheless, a myriad of studies have reported the variations of the branching pattern of the axillary artery [2-4].

In the palmar side of the hand, the ulnar artery ends by anastomosing with the superficial palmar branch of radial artery (SPBRA). That forms the superficial palmar arch, which gives rise to three common palmar digital arteries. The radial artery, meanwhile, mainly continues to the deep palmar arch by combining with the deep branch of ulnar artery. Before continuing the deep palmar arch, the radial artery gives off two branches, the princeps pollicis and radialis indicis arteries [1]. 


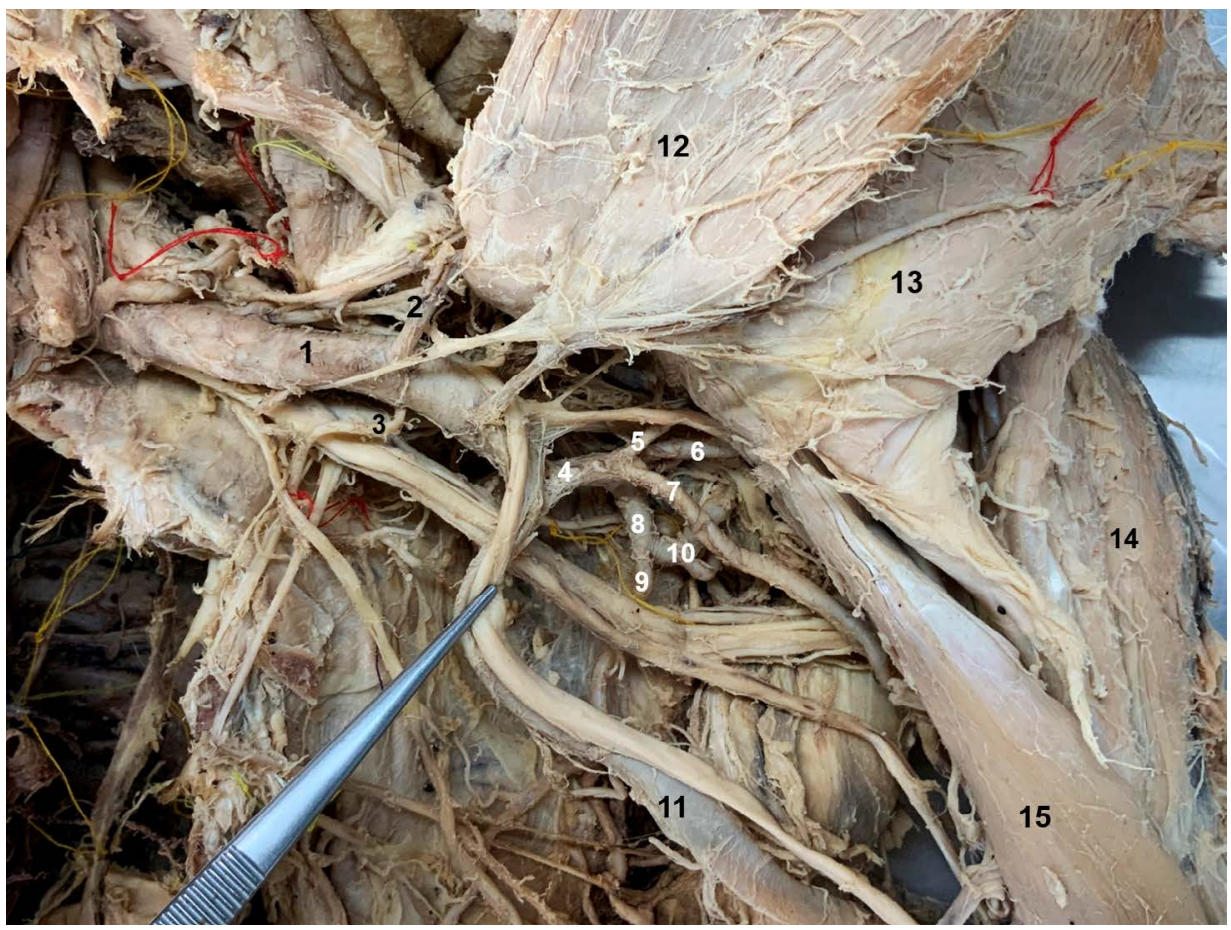

Fig. 1. Photograph of the left axillary artery. An aberrant common trunk arose from the third part of the axillary artery. 1, Axillary artery; 2, Thoraco-acromial artery; 3, Lateral thoracic artery; 4, Aberrant common trunk; 5, Anterior circumflex humeral artery; 6, Posterior circumflex humeral artery; 7, Deep brachial artery; 8, Subscapular artery; 9, Thoracodorsal artery; 10, Circumflex scapular artery; 11, Brachial artery; 12, Pectoralis minor; 13, Pectoralis major; 14, Deltoid; 15, Biceps brachii.

Various anomalous arterial patterns of the hand have been reported including the presence of the persistent median artery (PMA) [5-7], and the displacement of the superficial palmar arch $[8,9]$ Unusual arterial patterns are more vulnerable to iatrogenic injury during vascular transplantation or reconstruction procedures and may interfere with reliable interpretation of the angiographic images [10]. In this case report, a rare case of bilateral yet asymmetric variant arteries on the upper limb is described and their embryological backgrounds and clinical significance are discussed.

\section{CASE REPORT}

During a routine dissection in the gross anatomy class, the upper limb of an 84-year-old female cadaver was exposed, and the arterial branches were identified. This female cadaver had no specific medical history in both upper limbs. Out of 20 cadavers dissected from 2017 to 2019, it was the only case that had bilateral arterial variations in both arms and hands concurrently. The photos were taken by digital camera and illustrations were made by Adobe Photoshop (Adobe System Inc., San Jose, CA, USA). Herein, we delineate in the order of the left axilla, right axilla, PMA in the left hand, and large and subcutaneous SPBRAs in both hands with regard to arterial variations.

\section{Left axillary artery with high origin of the deep brachial artery}

An aberrant common trunk arose at the third part of left axillary artery and it divided into four branches including the anterior and posterior circumflex humeral artery, subscapular artery, and deep brachial artery (Fig. 1). The deep brachial artery traversed through the radial groove accompanying with the radial nerve. The axillary artery located typical position among the brachial plexus and continued as brachial artery, which showed normal anatomy in the arm.

\section{Right axillary artery variation with a common trunk at the second part}

A large common trunk arose at the second part of right axillary artery and gave off four branches including lateral 


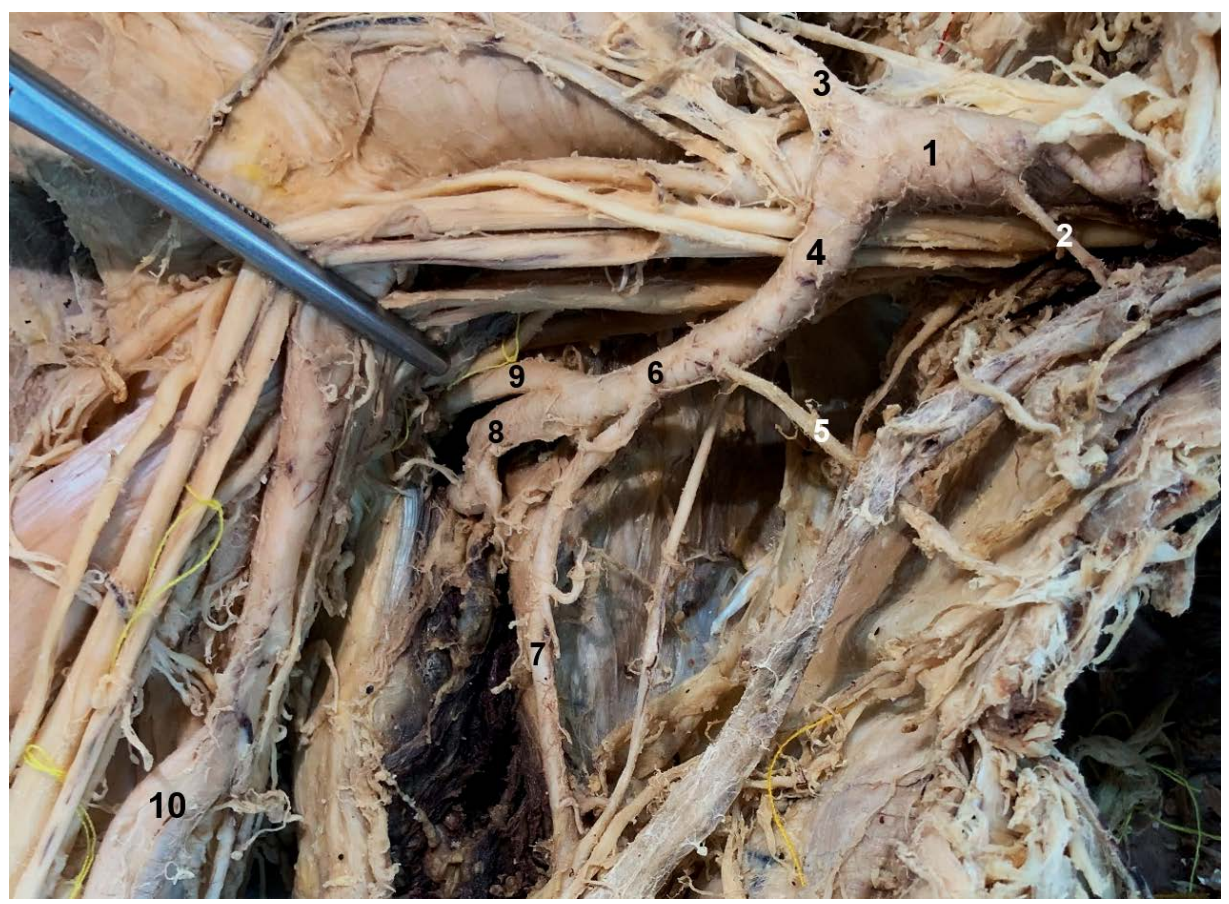

Fig. 2. Photograph of the right axillary artery. A large common trunk arose from the second part of the axillary artery, which was divided into four branches. 1, Axillary artery; 2, Superior thoracic artery; 3, Thoraco-acromial artery; 4, Aberrant common trunk; 5, Lateral thoracic artery; 6, Subscapular artery; 7, Thoracodorsal artery; 8 , Circumflex scapular artery; 9 , Common trunk of circumflex humeral artery; 10, Brachial artery.

thoracic artery, subscapular artery, and common stem of the posterior and anterior circumflex humeral artery (Fig. 2). The continuing axillary artery did not give off any other branches at the third part and continued as brachial artery.

\section{Persistent median artery in the left hand}

The PMA emerged from the anterior interosseous artery at the middle of the left forearm and coursed accompanying within the neurovascular sheath of the median nerve. PMA and median nerve traversed through the carpal tunnel with the median nerve without any other branches, and terminated by anastomosing with the superficial palmar arch (Fig. 3).

\section{Aberrant course of SPBRA in both hands}

The SPBRAs in both hands lay superficial to the abductor pollicis brevis (APB) to make the superficial palmar arch, unlike the normal course which run between the opponens pollicis and the APB (Arrowheads in Figs. 3 and 4). In addition, SPBRAs gave rise to a common trunk of the princeps pollicis and radialis indicis arteries, which are normally arising from the radial artery near the anatomical snuffbox immediately before it continues into the deep palmar arch (Arrows in Figs. 3 and 4).

\section{DISCUSSION}

In the present case, the bilateral yet asymmetric common trunk was observed in the second part of the right axillary artery, and in the third part of the left axillary, respectively. And the SPBRAs ran superficial to the thenar muscles on both hands with direct branches including the princeps pollicis and radialis indicis arteries. Moreover, the PMA was traversed with median nerve and formed the superficial palmar arch in concert with the radial and ulnar arteries on the left hand. These multiple variations encountered in a single cadaver are thought to be very rare over the previously reported anomalies of the upper limb.

In general, anatomical variations of the axillary artery are relatively common. There have been efforts to sort out branching patterns of the axillary artery, as well as the subclavian artery $[3,4,11]$. Astik et al. reported the incidence of variations in the arterial branching pattern of the upper limb 

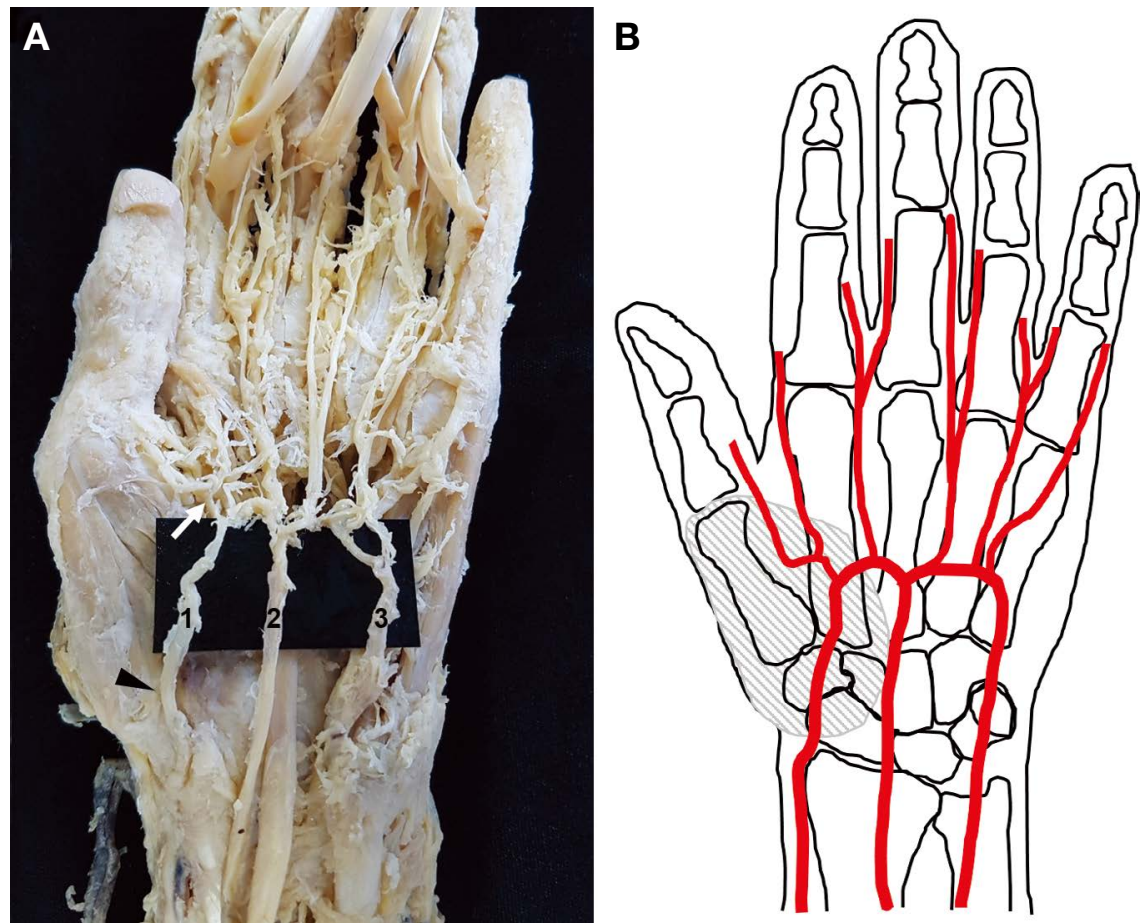

Fig. 3. Photograph of palmar surface of the left hand(A), and simplified illustration(B). Superficial palmar arch is formed from the ulnar artery, PMA, and SPBRA. The arrow shows a common trunk of the princeps pollicis and radialis indicis arteries. . Note the SPBRA(arrowhead) runs superficial to the thenar muscles, which is represented as shaded area in (B). 1, SPBRA; 2, PMA; 3, Ulnar artery.
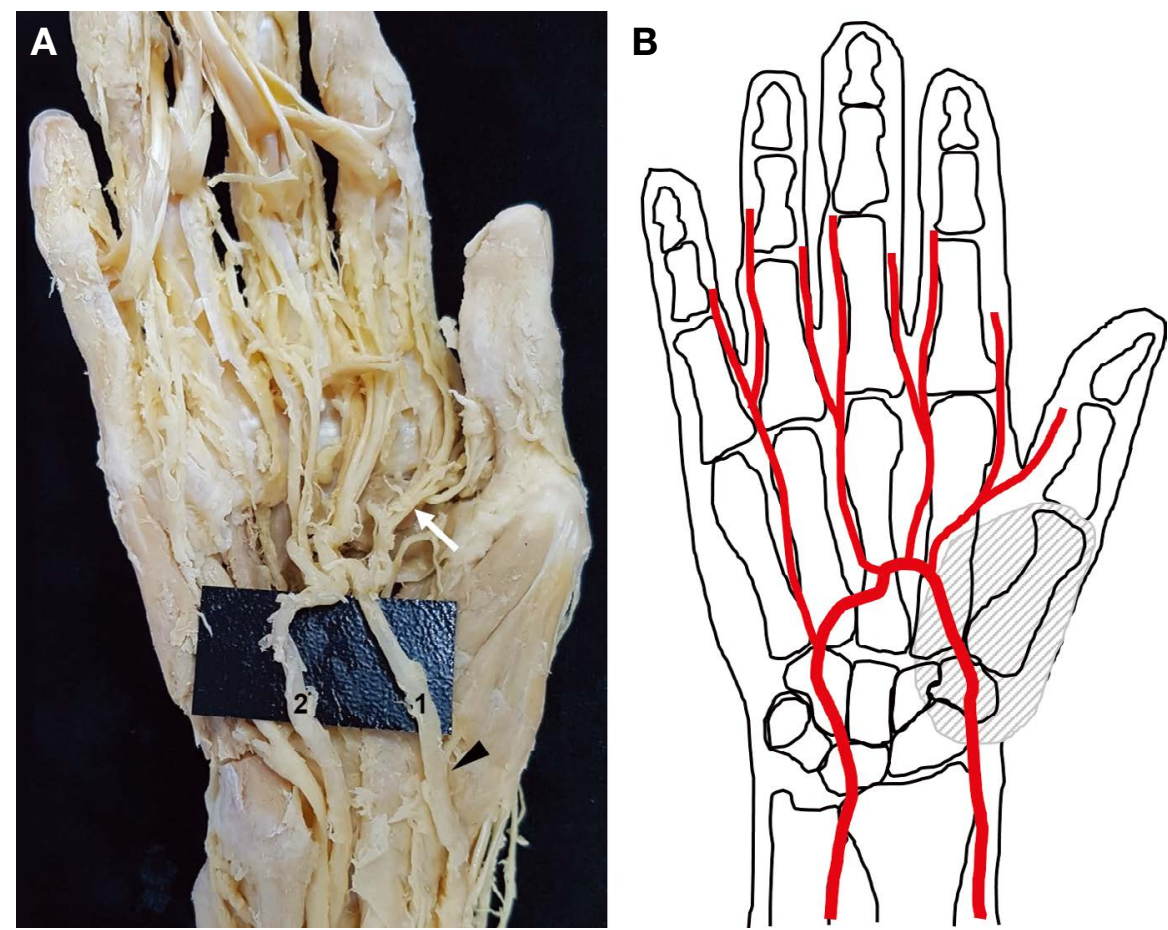

Fig. 4. Photograph of palmar surface of the right hand (A), and simplified illustration(B). The arrow shows a common trunk of the princeps pollicis and radialis indicis arteries. Note the SPBRA (arrowhead) runs superficial to the thenar muscles, which is represented as shaded area in (B). 1, SPBRA; 2, Ulnar artery. 
was $62.5 \%$, and a common trunk from the third part of the axillary arteries in $14.7 \%$ of the cases [2]. It has also been reported that the bilateral double axillary arteries which were divided at the second part, and deep brachial artery had a high origin at the third part [4]. Nevertheless, present case differs from other previous reports in that the common trunk of both axillary arteries arose around the pectoralis minor and the distribution pattern was asymmetric.

So far, there have been many reports of PMA, but its incidence was highly variable, ranging from 1.1 to $20 \%[5,6,12]$. This may be due to the difference between the number of cadavers investigated and the method of investigation. In addition, Feigl and colleagues showed that only $0.4 \%$ of cases in which PMA extends to the palm and forms a superficial palmar arch with radial and ulnar arteries [10]. In this case, PMA was surrounded by the sheath of the median nerve and traversed under the carpal tunnel, and the bifid of the median nerve was not observed, so it is estimated that there was no pain or other symptoms. However, some literature reported that the PMA can cause pain and other symptoms such as carpal tunnel syndrome [14].

The patterning process of arterial branches in the upper limb commences when the capillary plexus transformed to the axial artery, positioning the axillary, brachial and anterior interosseous arteries in terms of proximal to distal, respectively [4]. Variations of the axillary artery in present case may be arose spontaneously by unusual choice of paths in the primitive capillary plexuses during development. On the other hand, the anterior interosseous artery gives rise to the median, ulnar, and radial arteries [13]. After this, the median artery usually regresses after the $8^{\text {th }}$ week of gestation and forms the ulnar and radial arteries during the late stage of fetus [1,9]. However, the median artery can occasionally remain after the birth and passes along the median nerve toward the digits of the hands. In the present case, the median artery may be failed regression and formed the complete superficial palmar arch with the other arteries as radio-mediano-ulnar type [10]. Also, superficial course of the SPBRA is related to a hemodynamic mechanism between deep and superficial arteries in the palmar side of hand [9]. The unusual course of the radial artery appears to be due to chance variations in these hemodynamic factors, and this variation may lead to regression of the deep part and persistence of the SPBRA. Since our case did not include deep palmar arch dissection, we could not analyze the size and morphology of the deep palmar arch.
Detecting arterial variations has an important clinical implications for surgical interventions [2,4,11]. Knowing about unusual branching patterns of the axillary artery is necessary during reconstructing the axillary artery after trauma, catheterizing or cannulating the axillary artery and treating the thrombosis of the axillary artery [4]. Also, the anatomical relationship of the SPBRA is important. Most of cases describing aberrant courses of the SPBRA is reported from cadaveric studies. Therefore, it may be inferred that abnormal positioning of the SPBRA does not cause clinical symptoms and no intervention is required. However, if the thenar compartment is injured, superficial course of SPBRA could lead severe bleeding and it can also cause partial ischemia in fingers when grasping object by delivering external compression [15]. Also, the PMA can cause anterior interosseous nerve syndrome which the median artery penetrates median nerve, it can also cause carpal tunnel syndrome $[7,14]$.

The present study examines concurrent occurrence of the aberrant common trunk of the axillary arteries, PMA, and superficial course of SPBRAs. To date, despite numerous reports on each topic, there have been no cases in which these three variations have been reported in a single case. Thus, obtained reports of complicated variations may serve as a useful guide for hand surgeons and clinical anatomists. Knowledge of this unusual arterial variations in upper limb may help a diagnosis in vasculature of the axillary region and hand.

\section{REFERENCES}

1. Standring S. Gray's anatomy. 41th ed. New York: Elsevier; 2016. pp. 218-24.

2. Astik R, Dave U. Variations in branching pattern of the axillary artery: a study in 40 human cadavers. J Vasc Bras. 2012; 11:12-7.

3. Park SB, Lee JH, Choi IJ, Choi WI, Jin SC. Variation of the subscapular artery according to branching pattern of the axillary artery. Korean J Phys Anthropol. 2017;30:71-6.

4. Jurjus AR, Correa-De-Aruaujo R, Bohn RC. Bilateral double axillary artery: embryological basis and clinical implications. Clin Anat. 1999;12:135-40.

5. Buch C, Devora CM, Johnson LY, Rahimi OB, Kar R. Incomplete superficial palmar arch and bilateral persistent median artery. Int J Surg Case Rep. 2019;58:205-7.

6. Eid N, Ito Y, Shibata MA, Otsuki Y. Persistent median artery: 
cadaveric study and review of the literature. Clin Anat. 2011; 24:627-33.

7. Gassner EM, Schocke M, Peer S, Schwabegger A, Jaschke W, Bodner G. Persistent median artery in the carpal tunnel: color doppler ultrasonographic findings. J Ultrasound Med. 2002;21:455-61.

8. Onderoglu S, Basar R, Erbil KM, Cumhur M. Complex variation of the superficial palmar arch-case report. Surg Radiol Anat. 1997;19:123-5.

9. Ritz M, Mahendru S, Smyth M, Lavoipierre A, Goldan O, Pacifico MD. Dynamic arteries of the hand. Surg Radiol Anat. 2009;31:279-82.

10. Feigl GC, Petrac M, Pixner T, Ulz H, Mörth C, Dreu M. The superficial palmar arch and median artery as an example of misleading results due to a small number of investigated specimens or the use of different classifications. Ann Anat. 2012; 194:389-95.

11. Lee JY, Won DY, Oh SH, Hong SY, Woo RS, Baik TK, et al.
Three concurrent variations of the aberrant right subclavian artery, the non-recurrent laryngeal nerve and the right thoracic duct. Folia Morphol(Warsz). 2016;75:560-4.

12. Ikeda A, Ugawa A, Kazihara Y, Hamada N. Arterial patterns in the hand based on a three-dimensional analysis of 220 cadaver hands. J Hand Surg Am. 1988;13:501-9.

13. Rodriguez-Niedenfuhr M, Vázquez T, Parkin IG, Sañudo JR. Arterial patterns of the human upper limb: update of anatomical variations and embryological development. Eur J Anat. 2003;7:21-8

14. Dhal A, Prakash S, Kalra P, Khan Y. Unusual triad of bifid median nerve, persistent median artery, and aberrant muscle causing carpal tunnel syndrome: a case report. JBJS Case Connect. 2020;10:e1900468. doi: 10.2106/JBJS.CC.19. 00468 .

15. Singer G, Marterer R, Till H, Schmidt B. A rare anatomic variation of the superficial palmar branch of the radial artery causing pain. Surg Radiol Anat. 2018;40:349-52. 